change intervention by community volunteers for 12 months. A baseline, midterm and follow-up study were carried out during entire period of this intervention.

Results After 1 year of community based intervention, knowledge on iron richer food increased and causes of anaemia decreased from $62.9 \%$ to $82.2 \%$ and $86.1 \%$ to $71.5 \%$ respectively. Knowledge on symptoms and ways to prevent anaemia has also increased. It was found that more than two third (78.7\%) of participants has the knowledge on number of iron tablets required per month. After the intervention ended around $16.7 \%$ adolescents were taking iron tablet regularly meanwhile when iron folate supplementation was distributed free of cost and after that when it was sold door to door by the Community Volunteers (CVs) the rate was $94 \%$ and $28.5 \%$ respectively.

Conclusions Despite of the knowledge and positive attitude towards taking iron tablets, practice is not quite satisfactory, which might be due to nature of consumer and psychological behaviour along with inaccessibility of pharmacy is far away from the villages.

\section{P2-397 COLD SPELLS AND ASTHMA HOSPITALISATION IN NEW YORK STATE, USA: 1991-2006}

doi:10.1136/jech.2011.142976l.27

${ }^{1}$ E Fitzgerald, ${ }^{*}{ }^{2} \mathrm{C}$ Pantea, ${ }^{1,2} \mathrm{~S}$ Lin. ${ }^{1}$ University at Albany, School of Public Health, Rensselaer, New York, USA; ${ }^{2}$ New York State Department of Health, Center for Environmental Health, Troy, New York, USA

Introduction To help assess morbidity associated with cold spells, a study was conducted of cold temperature and asthma hospitalisations in New York State, USA.

Methods All hospital discharges among New York State residents with a diagnosis of asthma from November 1 to April 30 were obtained for 1991 to 2006. Temperatures were collected from stations in 13 weather regions in New York State. Universal Apparent Temperature was used to take into account wind speed and humidity, and a cold spell as defined as three consecutive days with a mean Universal Apparent Temperature $<$ the monthly $10^{\text {th }}$ percentile for each region. Percent change in asthma hospitalisation during and up to 4 days after each cold spell was evaluated using time series with Generalised Additive Models adjusting for temporal trends.

Results On a statewide basis, the results indicated that asthma hospitalisations decreased during cold spells for December through March by $4.84 \%$ (95\% CI -7.70 to -1.89 ). After cold spells, there was no change in the asthma hospitalisations for December through March, but hospitalisations increased in November $(9.98 \%, 95 \%$ CI 5.84 to 14.27$)$ and April (4.99\%, 95\% CI 1.18 and 8.94). The point estimates for the winter decrease and the November/April increase were greatest for the colder regions.

Conclusion The findings suggest that asthmatics may have difficulty acclimating to cold during the transitional months immediately before and after winter; in contrast, during a winter cold spell they may spend more time indoors, thereby preventing exacerbations.

\section{P2-398 FACTORS ASSOCIATED WITH MULTI-DRUG RESISTANT TUBERCULOSIS IN BANGLADESH}

doi:10.1136/jech.2011.142976l.28

${ }^{1} \mathrm{M}$ S Flora, ${ }^{*}$ M Nurul Amin, ${ }^{1} \mathrm{M}$ R Karim, ${ }^{2} \mathrm{~S}$ Afroze, ${ }^{1} \mathrm{~S}$ Islam, ${ }^{1} \mathrm{M}$ Ashraful Alam, ${ }^{1} \mathrm{M}$ Motahar Hossain. ${ }^{1}$ National Institute of Preventive and Social Medicine (NIPSOM), Dhaka, Bangladesh; ${ }^{2}$ Northern Medical College, Dhaka, Bangladesh

Introduction Despite success in tuberculosis control, multi-drug resistance tuberculosis (MDR-TB) in Bangladesh is increasing and currently MDR-TB rate is $3.6 \%$ in new cases and $19 \%$ in re-treatment cases. This study focused determination of environmental and host factors of MDR-TB which is warranted for effective prevention strategy.

Methods A case control study was conducted between January and August 2010. Purposively recruited 136 culture-proved MDR-TB cases and 152 cured TB patients were interviewed and $10 \mathrm{cc}$ of blood samples were taken. Associations between exposure and outcome variables were initially tested by $\chi^{2}$-test, t-test, ANOVA. A result was considered significant at $\mathrm{p}$ value $<0.05$. Effects of exposure variables were also assessed after adjusting for other variables by binary logistic regression models. Crude and adjusted OR with $95 \%$ CI was computed.

Results Younger age $(p=0.008)$, peri-urban locality $(p=0.002)$ associated with MDR-TB. History of contact $(p<0.001)$ and tuberculosis in the past $(p<0.001)$ were four and eight times, respectively, more likely to influence MDR-TB. Regularity [OR $0.05 ; 95 \%$ CI $(0.01$ to $0.39)$ ] and always observation of treatment [OR 0.25 ; 95\% CI $(0.10$ to 0.61$)$ ], sputum conversion [OR $0.02 ; 95 \% \mathrm{CI}(0.01$ to 0.08$)$ ] negatively associated with MDR-TB. Gender, socio-economic status and overcrowding did not show any influence. None was HIV positive, but its risk factors were more common in MDR-TB cases. Sputum conversion was the best predictor.

Conclusion Like other developing countries previous treatment status is the most important exposure variable. Strengthening of control activities might contribute in preventing development of resistance in tuberculosis patients.

\section{P2-399 WITHDRAWN}

\section{P2-400 WITHDRAWN}

\section{P2-401 EFFECT OF THE AGE, GENDER AND DAY OF THE WEEK ON ULTRA-PROCESSED FOOD CONSUMPTION}

doi:10.1136/jech.2011.142976l.31

${ }^{1,2}$ S C Fuchs, ${ }^{*}$ S L Rossato, ${ }^{1,2}$ L B Moreira, ${ }^{1}$ R L Henn, ${ }^{2}$ F D Fuchs. ${ }^{1}$ Postgraduate Studies Program in Epidemiology, Universidade Federal do Rio Grande do Sul, Porto Alegre, Rio Grande do Sul, Brazil; ${ }^{2}$ Hospital de Clinicas de Porto Alegre, Porto Alegre, Rio Grande do Sul, Brazil

Introduction The consumption of the ultra-processed food items has emerged as a concern in public health.

Objective To assess the effect of gender, age and day of the week on consumption of ultra-processed food items among adolescents and adults from southern Brazil.

Methods A cross-sectional study investigated a population-based sample of 234 individuals, aged 11-90 years. Dietary data was obtained by $24-\mathrm{h}$ recall. The food items were aggregated according to the level of processing as minimally (group 1), culinary or industry (group 2), and ultra-processed foods (group 3). The days of the week were categorised in weekend (Saturday and Sunday), beginning (Monday and Tuesday) and middle of the week (Thursday and Friday). The analysis was based on Generalised Estimated Equation (GEE).

Results Women consumed food items on average of 2.71 and men 3.22 times per day $(p=0.007)$. The positive effect of age was observed for all processed foods. The number of food items of group 1 consumed for adolescents was $60 \%$ lower than for older individuals. On the other hand, adolescents consumed $40 \%$ more ultraprocessed foods than older adults $(p<0.001)$. Food items of group 3 were more consumed in the middle of the week (4.86 times per day; $\mathrm{p}=0.040$ ) and by adolescents.

Conclusion The results show that ultra-processed foods consumed on weekends and by adolescents were an important problem of public health. 